\title{
Stereotactic body radiotherapy-induced abscopal effect twice after pembrolizumab failure in hereditary leiomyomatosis and renal cell carcinoma: a case report with genetic and immunologic analysis
}

\author{
Xinyue Zhang ${ }^{1}$, Yijun Zhang ${ }^{2}$, Yang Liu ${ }^{1}$, Yuanzhong Yang ${ }^{2}$, Pei Dong ${ }^{3}$, Liru He ${ }^{1}$, Fangjian Zhou ${ }^{3}$ \\ ${ }^{1}$ Department of Radiation Oncology, Sun Yat-sen University Cancer Center, State Key Laboratory of Oncology in South China, Collaborative \\ Innovation Center for Cancer Medicine, Guangzhou, China; ${ }^{2}$ Department of Pathology, Sun Yat-sen University Cancer Center, State Key \\ Laboratory of Oncology in South China, Collaborative Innovation Center for Cancer Medicine, Guangzhou, China; ${ }^{3}$ Department of Urology \\ Oncology, Sun Yat-sen University Cancer Center; State Key Laboratory of Oncology in South China, Collaborative Innovation Center of Cancer \\ Medicine, Guangzhou, China \\ Correspondence to: Liru He. Department of Radiation Oncology, Sun Yat-sen University Cancer Center, State Key Laboratory of Oncology in South \\ China, Collaborative Innovation Center for Cancer Medicine, No. 651, Dongfeng Road East, Guangzhou 510060, China. Email: helir@sysucc.org.cn; \\ Pei Dong. Department of Urology Oncology, Sun Yat-sen University Cancer Center, State Key Laboratory of Oncology in South China, \\ Collaborative Innovation Center for Cancer Medicine, No. 651, Dongfeng Road East, Guangzhou 510060, China. Email: dongpei@sysucc.org.cn.
}

\begin{abstract}
Hereditary leiomyomatosis and renal cell carcinoma (HLRCC) is a rare but aggressive disease. Immune checkpoint inhibitors (ICIs) have been an indispensable component for the management of advanced renal cell carcinoma, and stereotactic body radiotherapy (SBRT) has offered additional immunological effect boost for improving the treatment outcomes of the patients. However, the synergistic effect of ICIs with SBRT in HLRCC remains largely unexplored. We present the case of a 34-year-old woman with advanced HLRCC who underwent radical nephrectomy but soon relapsed at the retroperitoneal lymph nodes (RPLN). She was prescribed sunitinib but still progressed on twenty cycles with bulky RPLN and ascites. She was then treated with axitinib and pembrolizumab for twenty months, and received SBRT for the progression of RPLN upon which the first abscopal effect was observed via significant shrinkage of in-field and out-field tumor lesions. Five months later, she underwent a second course of SBRT for pelvic mass progression and the second abscopal effect was observed. Genetic and immunologic characteristics revealed a large number of tumor-infiltrating immune cells and high levels of PD-L1 expression. This case report demonstrates the synergistic effect of ICIs and SBRT in HLRCC and the potential mechanism for the repeated SBRT-induced abscopal effect, supporting the application of SBRT to oligometastatic lesion during ICIs treatment to delay disease progression. Further studies are needed to verify the strategy of combining ICIs and SBRT in advanced HLRCC.
\end{abstract}

Keywords: Pembrolizumab; stereotactic body radiotherapy (SBRT); abscopal effect; hereditary leiomyomatosis and renal cell cancer (HLRCC); case report

Submitted Jul 22, 2021. Accepted for publication Oct 29, 2021.

doi: 10.21037/tau-21-644

View this article at: https://dx.doi.org/10.21037/tau-21-644 


\section{Introduction}

Hereditary leiomyomatosis and renal cell carcinoma (HLRCC) is a rare type of kidney cancer that is typically associated with skin leiomyoma and uterine fibroids. It is characterized by germline mutation of fumarate hydratase $(\mathrm{FH})$, and often recur and metastasis early after radical nephrectomy (1).

At present, the NCCN guidelines recommend enrollment in clinical trials or sunitinib as the first-line systemic treatment for non-clear cell renal cell carcinoma (nccRCC) including HLRCC. However, the efficacy of sunitinib remains unsatisfactory with an objective response rate of only $9-11 \%$ and a progression-free survival (PFS) of only $5-6$ months $(2,3)$. The combination of bevacizumab with erlotinib is another possible regimen with a reported median PFS of 21.1 months and a manageable adverse event (AE) profile (grade $\geq 3 \mathrm{AE}: 49 \%$ ) from a phase II trial, in which $79 \%$ (34/43) of the HLRCC patients were first-line treated (4). The treatment experience of refractory HLRCC is even less.

Recently, two small sample-sized $(\mathrm{n}=10$ and $\mathrm{n}=16)$ studies reported high positivity (80-90\%) of PD-L1 expression in HLRCC; suggesting the potential application of immune checkpoint inhibitors (ICIs) $(5,6)$. As a boost to improve immunotherapeutic outcomes, stereotactic body radiotherapy (SBRT) has been suggested as an ideal partner in some solid tumors $(7,8)$. A pooled analysis of two randomized trials on metastatic non-small cell lung cancer (NSCLC) reported that the application of radiotherapy with pembrolizumab was associated with an improvement in median OS from 8.7 months to 19.2 months compared to pembrolizumab alone, without significant increase in toxicity (9). However, little is known about the effect of ICIs with SBRT in HLRCC. In this report, we describe a refractory HLRCC case who responded unexpectedly well to pembrolizumab followed by SBRT. Next-generation sequencing (NGS) and immunohistochemical staining (IHC) of the primary and metastatic tumor samples were performed to explore the immunologic characteristics.

We present the following article in accordance with the CARE reporting checklist (available at https://dx.doi. org/10.21037/tau-21-644).

\section{Case presentation}

A 34-year-old Chinese woman complaining of an increasingly right-sided lumbago after she discovered a

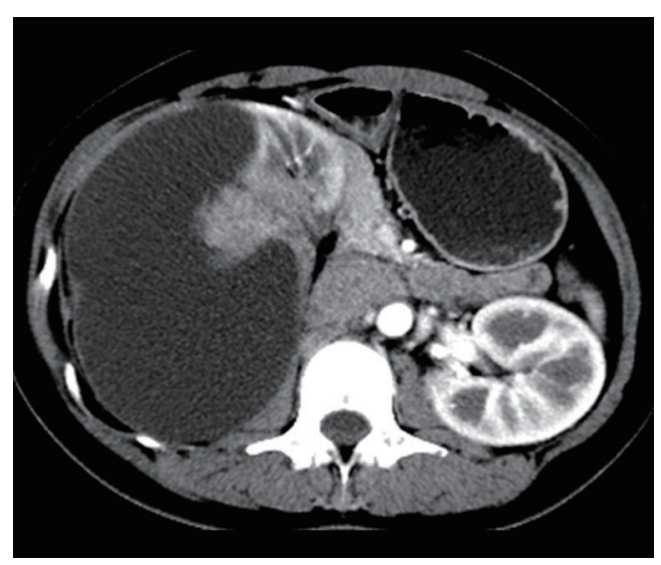

Figure 1 Computed tomography scan at baseline (December $4^{\text {th }}$, 2012) showing a $12.2 \mathrm{~cm} \times 7.9 \mathrm{~cm} \times 13.2 \mathrm{~cm}$ mass with solid and cystic components on the right kidney.

palpable right lumbar mass since November 2012. After admission, computed tomography (CT) imaging showed a $12.2 \mathrm{~cm} \times 7.9 \mathrm{~cm} \times 13.2 \mathrm{~cm}$ mass in her right kidney with enlarged retroperitoneal lymph nodes (RPLNs) in close proximity to major renal and abdominal blood vessels, without any distant metastasis (Figure 1) and multiple uterine fibroids were identified on PET-CT. On December 7, 2012, she underwent radical right-sided nephrectomy with RPLNs dissection and was pathologically diagnosed as pT2N1M0 nccRCC with the morphological feature of type 2 papillary renal cell carcinoma without adjuvant therapy. The timeline of this case is shown in Figure 2.

Eight months (September 1 $7^{\text {th }}, 2013$ ) after the nephrectomy, a follow-up CT scan observed relapse at the RPLNs and right-side psoas muscle metastasis. Sunitinib (50 mg QD, 4 weeks on, 2 weeks off) was prescribed and she achieved partial response (PR). Twenty-eight months (January $\left.1^{\text {st }}, 2016\right)$ later, she gradually developed ascites, characterized by abdominal distention and shortness of breath, which were not improving despite secondline treatment with axitinib (5 mg, QD) for 3 months. Subsequently, she received pembrolizumab (20 mg Q2W with cytokine-induced killer cells) concurrent with axitinib. As a result, a significant reduction in ascites was observed and she restarted working in June 2016. However, due to economic issues, she stopped pembrolizumab in January 2017 and the RPLN progressed shortly after two weeks. She restarted pembrolizumab (20 mg Q2W) from July 2017 and achieved PR again (Figure 3). In December 2017, she stopped pembrolizumab because of disease progression 


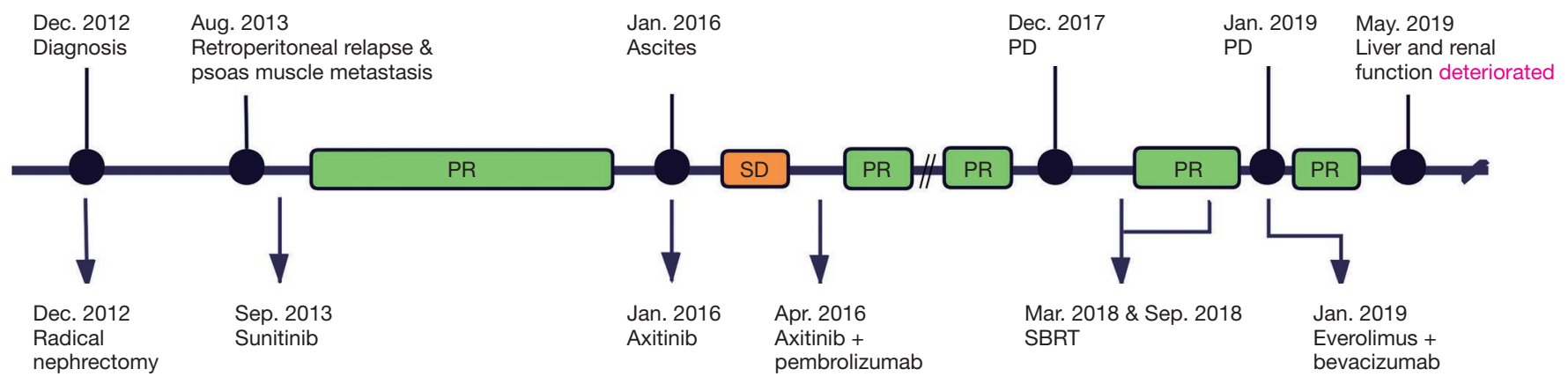

Figure 2 Timeline of the patient's treatment and clinical responses.

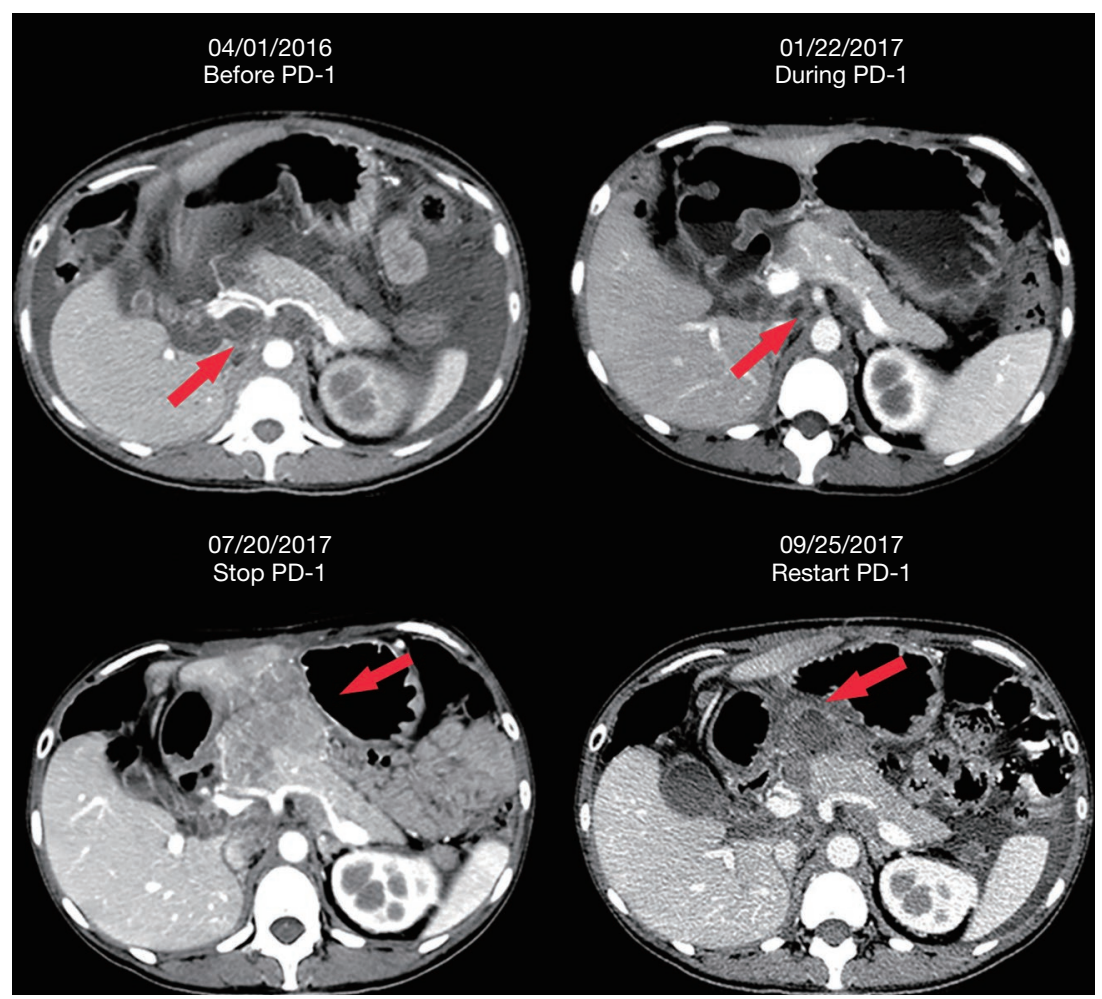

Figure 3 Response of RPLNs (red arrows) after pembrolizumab with axitinib. PR was observed after 10.5 months of pembrolizumab with axitinib. Progression was observed when the treatment was stopped for 6 months, and PR was observed again 2 months after the treatment was restarted. RPLNs, retroperitoneal lymph nodes; PR, partial response.

identified by two enhanced mass lesions at the splenic hilum and subcutaneous abdominal wall.

To identify a potentially effective regimen, we used the formalin-fixed paraffin-embedding (FFPE) tissue of primary renal sample for NGS and FFPE tissue of the metastatic abdominal wall mass for targeted sequencing (the methods can be seen in Appendix 1). $F H$ gene germline heterozygous mutation was found both in the primary and metastatic samples, and she was diagnosed as HLRCC. High tumor mutational burden score (11.05 vs. $0.76 \mathrm{mut} / \mathrm{Mb}$ ) and a high PTEN gene somatic mutation frequency (51.04\% vs. none) were also observed in the post-treated metastatic samples but not in the pre-treated primary samples (Figure 4). A followup CT scan revealed that her current main tumor lesion was still the retroperitoneal mass (RPLN fusion, $8.0 \mathrm{~cm}$ $\times 6.1 \mathrm{~cm}$ ) and radiotherapy was recommended after 


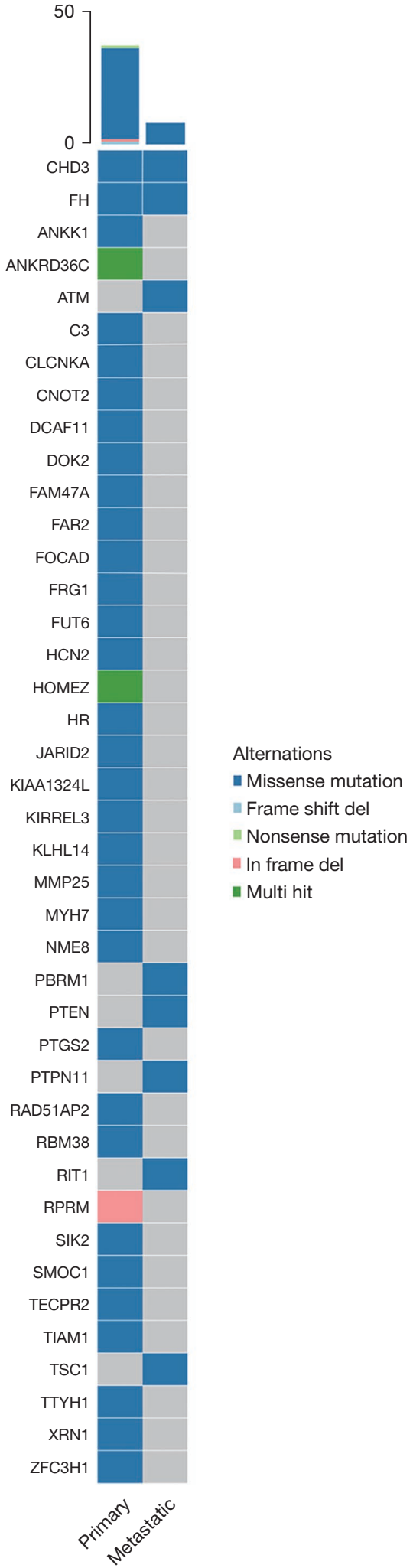

Figure 4 Waterfall plot of the most mutated genes in the primary and metastatic tumor samples. multidisciplinary team discussion in February 2018.

In March 2018, she received SBRT for the retroperitoneal mass with a total dose of $30 \mathrm{~Gy}$ in 6 fractions, and 2 months later (May 21 $1^{\text {st }}, 2018$ ) both the in-field and out-field tumors dramatically shrunk (Figure 5A). In August 2018, the follow-up CT scan revealed a new pelvic metastasis, then a subsequent SBRT (30 Gy/6 F) was given for the pelvic mass, and again 2 months later (November $1^{\text {st }}, 2018$ ) she achieved PR for both the in-field and out-field tumors (Figure 5B). However, after the second SBRT in January 2019, multiple new metastases were observed.

To determine possibly effective drugs, a mini patientderived xenograft (MiNiPDX), was constructed (the method can be seen in Appendix 1) and a combination of everolimus and bevacizumab was identified as the most effective regimen (Figure 6). She was thereby given everolimus (10 mg, QD) and bevacizumab (200 mg, Q3W) from June $16^{\text {th }}, 2019$, and achieved PR. However, the regimen had to be suspended because her renal and liver function deteriorated, and she finally died from tumor progression and multiple organs failure on July 10, 2019.

After the patient's death, to further investigate the cause for her long 20-month good response to immunotherapy, we explored the characteristics of her tumor immune microenvironment (TIME) for a series of immune-related factors in her primary and metastatic tumor samples via IHC (Figure 7). All the antibodies for IHC and the method of analysis can be seen in Appendix 1. Our findings showed that this could be related to a high positivity of PD-L1 expression in her tumor cells (primary cells: CPS $=30$, metastatic cells: $\mathrm{CPS}=28$ ) and $\mathrm{CD} 8^{+} \mathrm{T}$ cells (primary: $82.22 \pm 28.33$ /high power field, metastasis: $78.89 \pm 13.47 /$ high power field), and negative expression of PD-1 and Foxp3 in the intratumoral and peritumoral areas of her primary and metastatic tumors.

All procedures performed in this study involving human participants were in accordance with the ethical standards of the institutional and/or national research committee(s) and with the Helsinki Declaration (as revised in 2013). Written informed consent was obtained from the parents of the patient for publication of this case report and accompanying images. A copy of the written consent is available for review by the editorial office of this journal.

\section{Discussion}

To our knowledge, this is the first case of HLRCC that demonstrated an unexpectedly good response to ICI-based 

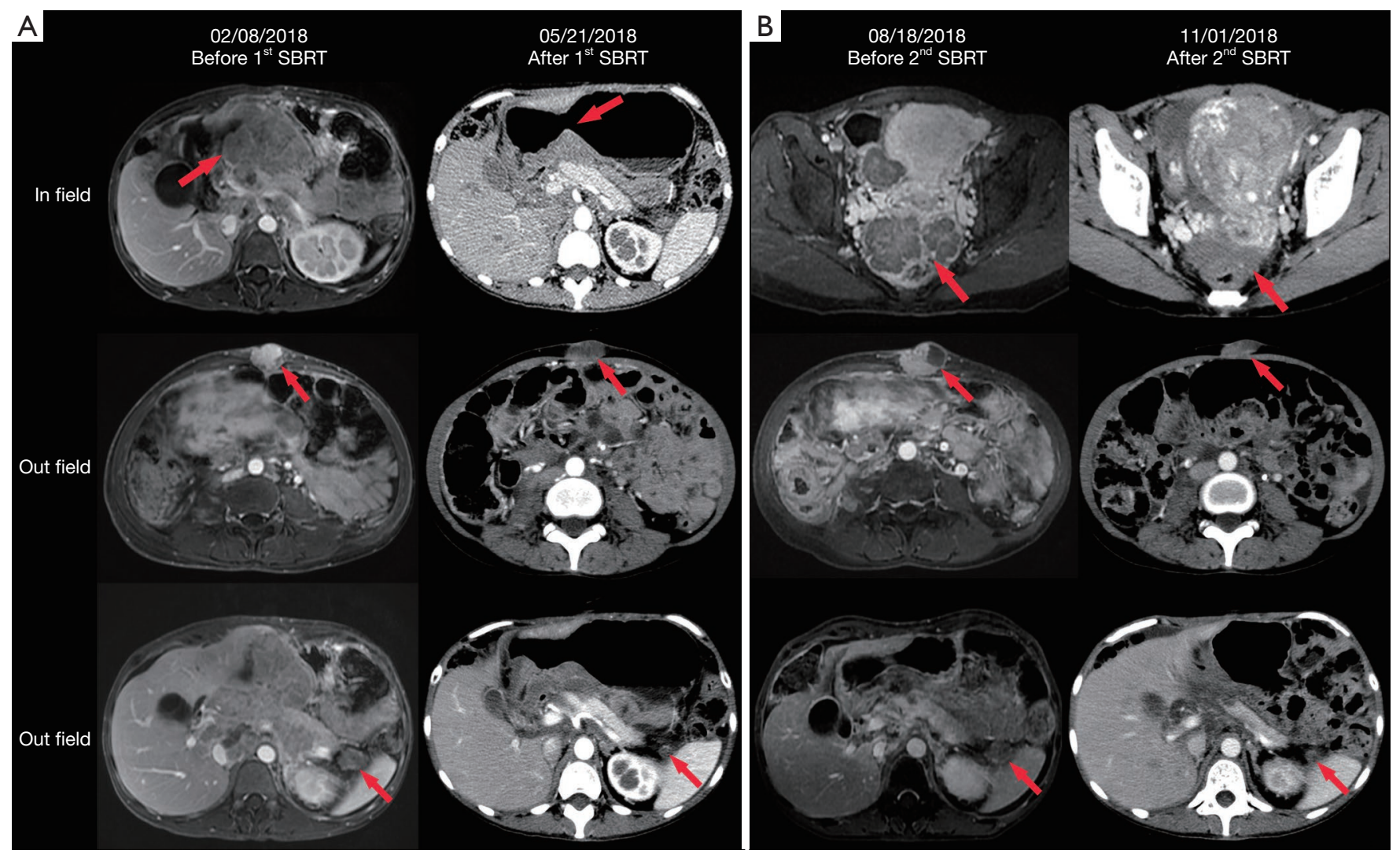

Figure 5 Twice shrinkage of in-field and out-field tumors after SBRT. (A) The images in the first line referred to a retroperitoneal metastasis (red arrows) measuring $8.1 \mathrm{~cm} \times 6.0 \mathrm{~cm}$ showing a significant reduction in size 3.5 months after the first course of SBRT. The images in the second and third lines referred to the first abscopal effect of the tumor lesions at the subcutaneous abdominal wall and splenic hilum (red arrows), respectively. (B) The images in the first line referred to the pelvic metastatic lesions (red arrows), with the larger one measuring $4.8 \mathrm{~cm}$ $\times 4.5 \mathrm{~cm}$, demonstrating remarkable shrinkage 2.5 months after the second course of SBRT. The images in the second and third lines referred to the second abscopal effect of the tumor lesions at the subcutaneous abdominal wall and splenic hilum (red arrows), respectively. SBRT, stereotactic body radiotherapy.

treatment followed by SBRT, with abscopal effect observed twice during the treatment period, in a patient with bulky relapsed HLRCC. HLRCC is a rare type of refractory disease without definitive consensus on its management. Although the guidelines have not yet recommended ICIs for HLRCC, there are data indicating the potential benefit of ICIs in such patients (10). Similar to the findings of Furuya et al. (7/10 cases; PD-L1 positivity: $2+)$ and Sun et al. (8/16 cases; PD-L1 expression $\geq 30 \%$ ), high expression of PD-L1 was also observed in our HLRCC case $(5,6)$. She achieved PR on ICI-based therapy and her lesions were controlled for 20 months; consistent with the observation by Sun et al. who found that for $\mathrm{FH}$-deficient metastatic renal cell carcinoma patients receiving ICIs based therapy $(n=6)$, their median PFS was significantly longer than those treated with antiangiogenic monotherapy ( $\mathrm{n}=12)$ (13.3 vs. 5.1 months) (6). In addition, the TIME of our case had a relatively high infiltration degree of $\mathrm{CD}^{+} \mathrm{T}$ cells without Foxp $3^{+}$Treg cells, indicating a favorable baseline microenvironment for ICI therapy. Taken together, we suggested that HLRCC characterized by high positivity of PD-L1 expression and $\mathrm{CD}^{+} \mathrm{T}$ cells infiltration could be a good candidate for ICIs therapy.

Despite some research on the underlying resistance mechanism of action of ICIs, however, this remains largely unclear $(11,12)$. Recently, somatic PTEN mutations were found to be associated with resistance to ICIs by altering immunosuppressive environments in glioblastoma (13). $P T E N$-mutant tumors were more likely to have elevated levels of $\mathrm{CD} 8^{+}$TAMs infiltration, which was associated 
A

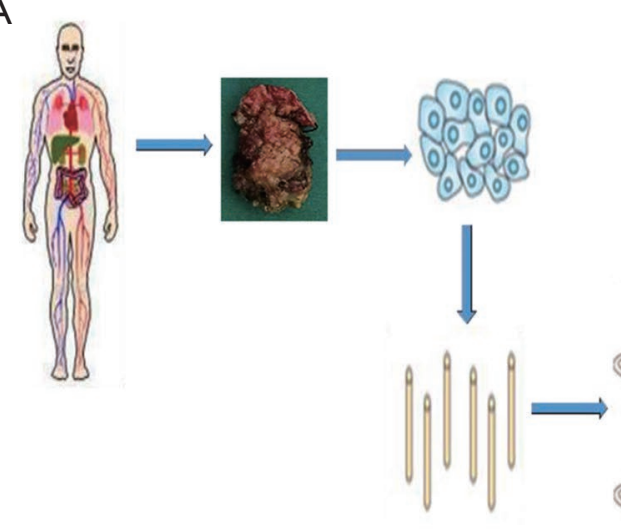

Experimental device
MiNiPDX flow chart

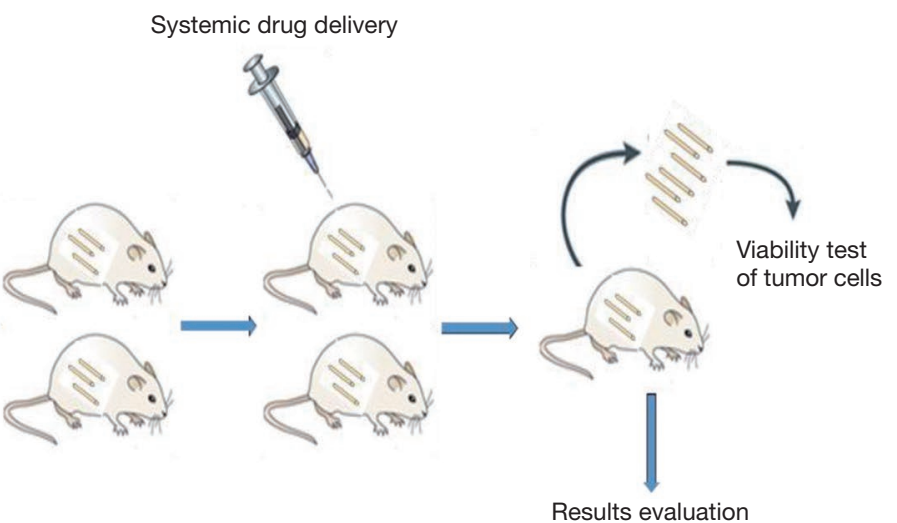

B

\begin{tabular}{|c|c|c|}
\hline & Drugs & Dosing \\
\hline N/A & \multicolumn{2}{|c|}{ No drug delivery } \\
\hline Regimen 1 & $\begin{array}{l}\text { Erlotinib }+ \\
\text { Bevacizumab }\end{array}$ & $\begin{array}{l}\text { Erlo, } 50 \mathrm{mg} / \mathrm{kg}, \mathrm{PO}, \mathrm{QD}{ }^{\star} 7 \\
+ \text { Beva, } 10 \mathrm{mg} / \mathrm{kg}, \mathrm{IP}, \mathrm{Q} 4 \mathrm{D}^{\star 2}\end{array}$ \\
\hline Regimen 2 & $\begin{array}{l}\text { Everolimus }+ \\
\text { Bevacizumab }\end{array}$ & $\begin{array}{l}\text { Evero, } 10 \mathrm{mg} / \mathrm{kg}, \mathrm{PO}, \mathrm{QD}^{*} 5 \\
+ \text { Beva, } 10 \mathrm{mg} / \mathrm{kg}, \mathrm{IP}, \mathrm{Q} 4 \mathrm{D}^{\star 2}\end{array}$ \\
\hline Regimen 3 & $\begin{array}{l}\text { Lenvatinib + } \\
\text { Everolimus }\end{array}$ & $\begin{array}{l}\text { Lenva, } 100 \mathrm{mg} / \mathrm{kg}, \mathrm{PO}, \mathrm{QD}^{\star} 7 \\
+ \text { Evero, } 10 \mathrm{mg} / \mathrm{kg}, \mathrm{PO}, \mathrm{QD}^{\star} 5\end{array}$ \\
\hline \multicolumn{3}{|c|}{$\begin{array}{l}\text { Appendix: } \mathrm{IP}=\text { Intraperitoneal, } \mathrm{PO}=\text { oral, } \mathrm{QD}=\text { Once a day, } \mathrm{Q} 4 \mathrm{D}=\text { Once } \\
\text { every } 4 \text { days }\end{array}$} \\
\hline
\end{tabular}

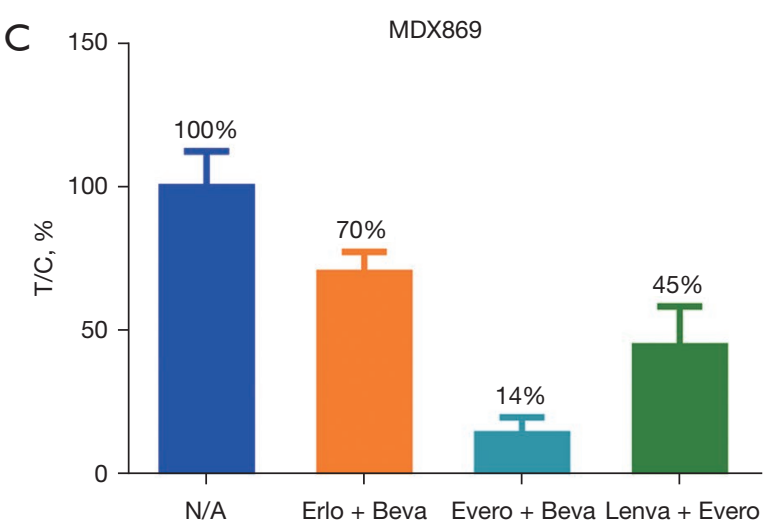

Figure 6 Experiment design and the result of MiNiPDX test. (A) MiNiPDX flow chart; (B) The regimens of the control group and the three treatment groups; (C) Relative proliferation rate of tumor cells under different regimens. T/C (\%) refers to the percentage of tumor cells proliferation in the treatment group relative to the control group. The lower the value, the stronger the inhibition effect on tumor cells. MiNiPDX, mini patient-derived xenograft. T/C $\%=\mathrm{T} / \mathrm{C} \times 100 \%$, the ratio of proliferation rate of tumor cells in treatment groups to control group.

with poor survival in glioblastoma (13). In the postICI failure metastatic tissues of the present case, a high frequency of somatic PTEN mutation was observed with a trend of elevated levels of CD $68^{+}$TAMs infiltration compared to those in pre-treated primary tumor sites, but no significant difference was observed. Collectively, these data indicated that PTEN mutations might be one of the important triggers for resistance to ICIs in human cancers, including HLRCC.

Renal cell carcinoma is generally believed to be radioresistant, however, the patient reported in this study had unexpected good sensitivity to radiotherapy. Although the prescribed radiation dose was relatively low, for avoiding damage of surrounding normal organs such as the small intestine, the in-field tumor shrunk dramatically only 2 months after the completion of SBRT. Recently, Ma et al. reported that the inhibition of nuclear PTEN tyrosine phosphorylation caused by PTEN mutation could enhance the radiosensitivity of glioma through attenuated DNA repair (14). Thus, we speculated that PTEN mutation (Figure 4), which was reported to be associated with ICIs resistance, might have also contributed to the radiosensitization of the present HLRCC case.

It was also very interesting to observe abscopal effects induced by SBRT twice even after progression on pembrolizumab, referring to the regression of metastatic tumors at distant sites that were not irradiated. SBRT is well accepted as the optimal partner of immunotherapy, acting as an $i n$-situ vaccine for enhancing the systemic efficacy of immunotherapy (15). Besides, immunotherapy has been 
A
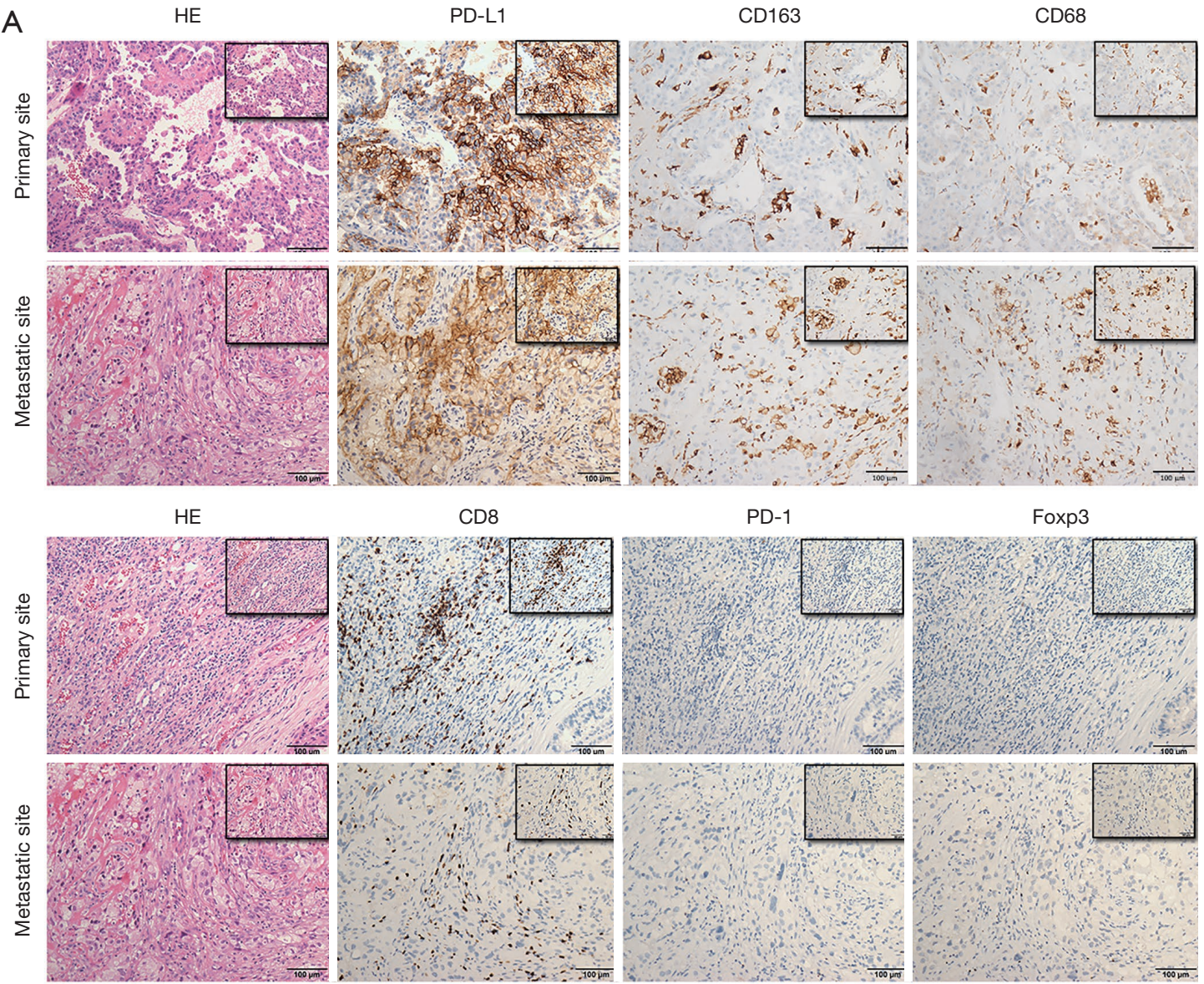

B
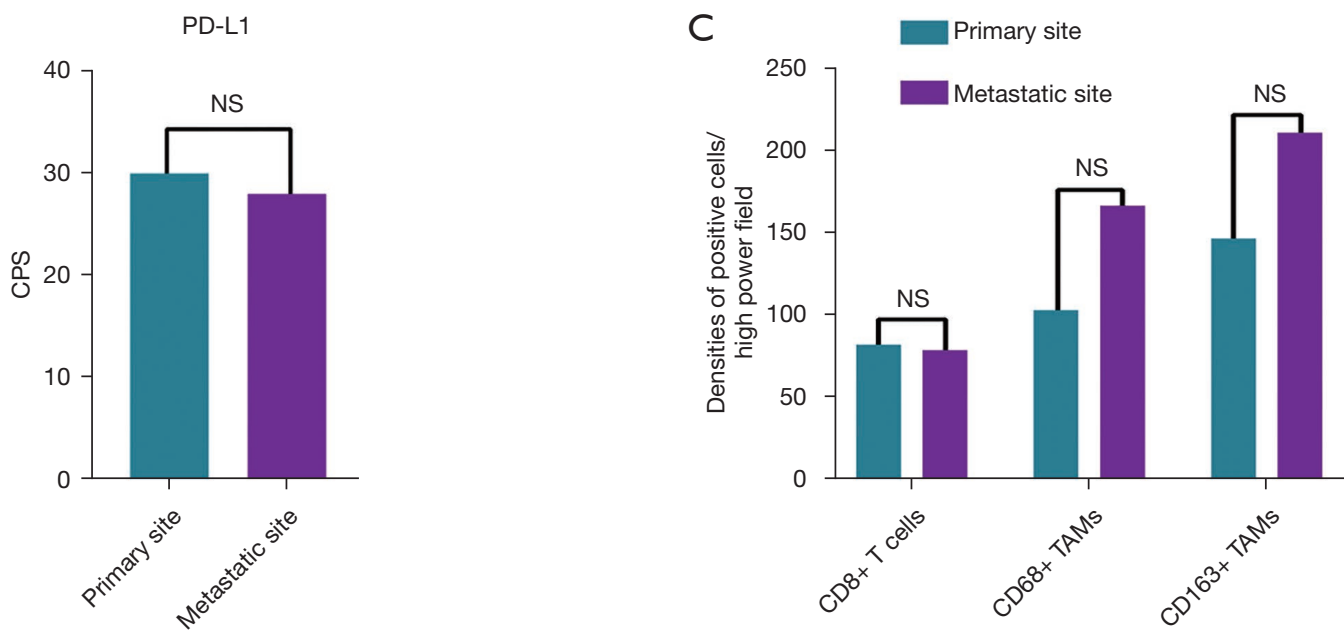

Figure 7 The tumor immune microenvironment characteristics at the primary and metastatic sites. (A,B) Representative hematoxylineosin staining images and IHC images of PD-L1 (Cell Signaling Technology, 13684), PD-1 (Ascend Biology, ZM43), FOXP3 (mAbcam, ab22510), CD8 (Ascend Biology, C8/144B), CD68 (ZSGB-BIO, ZM0464) and CD163 (ZSGB-BIO, ZM0428) at the primary and metastatic sites, magnification of $\times 200$ with $\times 400$ insert. (C) The CPS of PD-L1+ cells in primary and metastatic sites; (D) The densities of CD $8^{+} \mathrm{T}$ cells, CD68 ${ }^{+}$TAMs and CD163 ${ }^{+}$TAMs at the primary and metastatic sites. CPS, combined positive score; IHC, immunohistochemical staining; PD-L1, programmed cell death 1 ligand 1; PD-1, programmed cell death 1; TAMs, tumor-associated macrophages. 
applied to boost the abscopal effect, usually in concurrent or after SBRT $(7,15)$. However, the optimal timing of combining radiotherapy and immunotherapy is still unclear. A randomized phase 1 trial reported that concomitant rather than sequential SBRT with pembrolizumab in metastatic UC patients had better OS (12.1 vs. 4.5 months) (16). Recently, two patients with metastatic Merkel cell carcinoma that progressed on pembrolizumab were also reported to have an abscopal effect after a short course of radiation therapy by $\mathrm{Xu}$ et al. (17). In our case, the patient became resistant to pembrolizumab with axitinib after 20 months with multiple tumor progression. When adding SBRT to the main tumor lesion, irradiated tumor lesion achieved PR and non-irradiated tumor lesions regenerated an immune response which was the immunostimulatory effect of SBRT. These data suggested that SBRT may have synergistic effects with immunotherapy even in the setting of patients who were proven to be progressing on ICIs.

Based on this case, we theorize that when the first-time immunostimulatory effect of SBRT occurred, restarting ICIs treatment might be considered to maintain longterm positive feedback; supported by the observation that in melanoma, a proportion of patients had persistent regression of out-field lesions when ICI was continued after SBRT to a lesion that had progressed on ICI-based treatment (18). Also, as the liver and renal function of this patient progressively deteriorated after multimodal treatments, earlier application of supportive therapy such as interventional therapy and/or dialysis could be considered to either prevent or delay such outcomes.

\section{Conclusions}

Here, we report a refractory HLRCC case who had an initial good response to pembrolizumab combined therapy and abscopal effect induced by SBRT twice after progression on pembrolizumab. This case suggests that even low dose SBRT may synergistically work with ICIs in HLRCC, indicating that tumor-infiltrating immune cells and PD-L1 expression level might be an important factor to engage SBRT-induced abscopal effect. ICIs combined with SBRT might be a promising strategy for HLRCC, but further studies are needed to determine the effectiveness of this regimen in the clincal cohort and uncover the underlying mechanisms.

\section{Acknowledgments}

We would like to thank Dr. Seeruttun Sharvesh Raj for his help in polishing our paper.

Funding: This work was supported by a grant from the Nature Science Foundation of China (Grant number: No. 81772483).

\section{Footnote}

Reporting Checklist: The authors have completed the CARE reporting checklist. Available at https://dx.doi. org/10.21037/tau-21-644

Conflicts of Interest: All authors have completed the ICMJE uniform disclosure form (available at https://dx.doi. org/10.21037/tau-21-644). The authors have no conflicts of interest to declare.

Ethical Statement: The authors are accountable for all aspects of the work in ensuring that questions related to the accuracy or integrity of any part of the work are appropriately investigated and resolved. All procedures performed in studies involving human participants were in accordance with the ethical standards of the institutional and/or national research committee(s) and with the Helsinki Declaration (as revised in 2013). Written informed consent was obtained from the parents of the patient for publication of this case report and accompanying images. A copy of the written consent is available for review by the editorial office of this journal.

Open Access Statement: This is an Open Access article distributed in accordance with the Creative Commons Attribution-NonCommercial-NoDerivs 4.0 International License (CC BY-NC-ND 4.0), which permits the noncommercial replication and distribution of the article with the strict proviso that no changes or edits are made and the original work is properly cited (including links to both the formal publication through the relevant DOI and the license). See: https://creativecommons.org/licenses/by-nc-nd/4.0/.

\section{References}

1. Chen YB, Brannon AR, Toubaji A, et al. Hereditary leiomyomatosis and renal cell carcinoma syndromeassociated renal cancer: recognition of the syndrome by pathologic features and the utility of detecting aberrant succination by immunohistochemistry. Am J Surg Pathol 2014;38:627.

2. Tannir NM, Jonasch E, Albiges L, et al. Everolimus versus 
sunitinib prospective evaluation in metastatic non-clear cell renal cell carcinoma (ESPN): a randomized multicenter phase 2 trial. Eur Urol 2016;69:866-74.

3. Ravaud A, Oudard S, De Fromont M, et al. First-line treatment with sunitinib for type 1 and type 2 locally advanced or metastatic papillary renal cell carcinoma: a phase II study (SUPAP) by the French Genitourinary Group (GETUG). Ann Oncol 2015;26:1123-8.

4. Srinivasan R, Gurram S, Al Harthy M, et al. Results from a phase II study of bevacizumab and erlotinib in subjects with advanced hereditary leiomyomatosis and renal cell cancer (HLRCC) or sporadic papillary renal cell cancer. J Clin Oncol 2020;38:Abstract 5004.

5. Furuya M, Iribe Y, Nagashima Y, et al. Clinicopathological and molecular features of hereditary leiomyomatosis and renal cell cancer-associated renal cell carcinomas. J Clin Pathol 2020;73:819-25.

6. Sun G, Zhang X, Liang J, et al. Integrated Molecular Characterization of Fumarate Hydratase-deficient Renal Cell Carcinoma. Clin Cancer Res 2021;27:1734-43.

7. Liu Y, Dong Y, Kong L, et al. Abscopal effect of radiotherapy combined with immune checkpoint inhibitors. J Hematol Oncol 2018;11:1-15.

8. Zhuang $\mathrm{H}$. Abscopal effect of stereotactic radiotherapy combined with anti-PD-1/PD-L1 immunotherapy: Mechanisms, clinical efficacy, and issues. Cancer Commun (Lond) 2020;40:649-54.

9. Theelen WSME, Chen D, Verma V, et al. Pembrolizumab with or without radiotherapy for metastatic non-small-cell lung cancer: a pooled analysis of two randomised trials. Lancet Respir Med 2021;9:467-75.

10. Lavacchi D, Pellegrini E, Palmieri VE, et al. Immune Checkpoint Inhibitors in the Treatment of Renal Cancer:

Cite this article as: Zhang X, Zhang Y, Liu Y, Yang Y, Dong $\mathrm{P}, \mathrm{He}$ L, Zhou F. Stereotactic body radiotherapy-induced abscopal effect twice after pembrolizumab failure in hereditary leiomyomatosis and renal cell carcinoma: a case report with genetic and immunologic analysis. Transl Androl Urol 2021;10(11):4304-4312. doi: 10.21037/tau-21-644
Current State and Future Perspective. Int J Mol Sci 2020;21:4691.

11. Sharma P, Hu-Lieskovan S, Wargo JA, et al. Primary, Adaptive, and Acquired Resistance to Cancer Immunotherapy. Cell 2017;168:707-23.

12. Li SJ, Chen JX, Sun ZJ. Improving antitumor immunity using antiangiogenic agents: Mechanistic insights, current progress, and clinical challenges. Cancer Commun (Lond) 2021;41:830-50.

13. Cheng F, Eng C. PTEN mutations trigger resistance to immunotherapy. Trends Mol Med 2019;25:461-3.

14. Ma J, Benitez JA, Li J, et al. Inhibition of nuclear PTEN tyrosine phosphorylation enhances glioma radiation sensitivity through attenuated DNA repair. Cancer Cell 2019;35:504-18.e7.

15. Ngwa W, Irabor OC, Schoenfeld JD, et al. Using immunotherapy to boost the abscopal effect. Nat Rev Cancer 2018;18:313-22.

16. Sundahl N, Vandekerkhove G, Decaestecker K, et al. Randomized Phase 1 Trial of Pembrolizumab with Sequential Versus Concomitant Stereotactic Body Radiotherapy in Metastatic Urothelial Carcinoma. Eur Urol 2019;75:707-11.

17. Xu MJ, Wu S, Daud AI, et al. In-field and abscopal response after short-course radiation therapy in patients with metastatic Merkel cell carcinoma progressing on PD-1 checkpoint blockade: a case series. J Immunother Cancer 2018;6:43.

18. Ribeiro Gomes J, Schmerling RA, Haddad CK, et al. Analysis of the Abscopal Effect With Anti-PD1 Therapy in Patients With Metastatic Solid Tumors. J Immunother 2016;39:367-72. 


\section{Materials and methods}

\section{IHC and analysis}

IHC was performed to analyze the expression of PD-L1, PD-1, CD8, CD68, CD163 and Foxp3 in the primary and metastatic tumor tissues. Positive PD-L1 expression was scored using a CPS system based on the membrane-positive tumor cells and immune cells. $\mathrm{CPS}=[(\mathrm{PD}-\mathrm{L} 1+$ tumor cells $+\mathrm{PD}-\mathrm{L} 1+$ immune cells $) /$ total number of tumor cells $] \times 100$. The average density (cells/ high power field) of each lymphocyte subset was quantitatively scored by pathologist. Nine high power fields (magnification $\times 400$ ) in tumor areas were selected randomly for positive cells counting and the calculation of average number of cells. The antibodies used in the experiment were as follows: anti-PD-L1 (13684, Cell Signaling Technology), anti-CD8 (C8/144B, Ascend Biology), anti-PD-1 (ZM43, Ascend Biology), anti-Foxp3 (ab22510, mAbcam), anti-CD68 (ZM0464, ZSGB-BIO), anti-CD163 (ZM0428, ZSGB-BIO).

\section{NGS}

High-quality genomic DNA (gDNA) was extracted from the primary Formalin-fixed paraffin-embedded (FFPE) tumor tissue using commercial kits according to the manufacturer's instructions (Cat Qiagen, No. 56404). Libraries for Exome sequencing were prepared using the KAPA HyperPrep Kits (Roche, KK8504) and $x$ Gen ${ }^{\circledR}$ Exome Research Panel v1.0 (IDT). The final libraries were subjected to Illumina sequencing on the Illumina Novaseq6000 sequencer.

\section{Targeted sequencing analyses}

High-quality gDNA was extracted from the metastatic FFPE tumor tissues and peripheral blood mononuclear cells using DNeasy Blood \& Tissue Kit (Qiagen, Inc.) according to the manufacturer's instructions. The Panel used for targeted sequencing was HJiA_618G in Accel-NGS 2S DNA Library Kit (Swift Biosciences, Inc.). Samples underwent paired-end sequencing on an Illumina Novaseq platform (Illumina Inc) with a 150-bp read length. Mean coverage of $1260.5 \times$ and $223.6 \times$ were achieved for tumor gDNA and peripheral blood mononuclear cells gDNA, respectively.

\section{MiNiPDX}

MiNiPDX was performed in vivo using OncoVee ${ }^{\circledR}$ MiNiPDX for screening clinically effective regimens for this patient. In the test, patient-derived tumor cells were arrayed within hollow fiber capsules, implanted subcutaneously into mice and cultured for 7 days. The administration was started on the first day of inoculation. There were three regimens as followed: erlotinib plus bevacizumab, everolimus plus bevacizumab and lenvatinib plus everolimus. The cellular activity morphology and pharmacokinetics were systematically evaluated according to ATP test. Reponses to drug were examined by tumor cell relative growth inhibition rate after taking out the MiNiPDX devices. 